\title{
Carnets
}

Revue électronique d'études françaises de l'APEF

Deuxième série - 7| 2016

Plurilinguisme et migrations dans la littérature de langue française

\section{Une recherche qualitative sur les trajectoires migratoires des migrants}

Migratoires des migrants : du recueil des observables à l'analyse des données

\section{Abla Matalah}

\section{OpenEdition}

\section{Journals}

Édition électronique

URL : http://journals.openedition.org/carnets/1086

DOI : $10.4000 /$ carnets. 1086

ISSN : 1646-7698

Éditeur

APEF

Référence électronique

Abla Matalah, " Une recherche qualitative sur les trajectoires migratoires des migrants », Carnets [En ligne], Deuxième série - 7 | 2016, mis en ligne le 31 mai 2016, consulté le 21 avril 2019. URL : http:// journals.openedition.org/carnets/1086 ; DOI : 10.4000/carnets.1086

Ce document a été généré automatiquement le 21 avril 2019

Carnets est mis à disposition selon les termes de la licence Creative Commons - Atribution - Pas d'utilisation commerciale 4.0 International. 


\section{Une recherche qualitative sur les trajectoires migratoires des migrants}

Migratoires des migrants : du recueil des observables à l'analyse des données

Abla Matalah

\section{Introduction}

1 Le concept de Portfolio est à l'origine un outil utilisé dans le domaine artistique. Il se compose de certaines réalisations de l'artiste. Récemment, ce concept a été transposé dans le domaine scolaire avec l'objectif de stimuler l'apprentissage, de reproduire le parcours d'apprentissage formel et informel de tous les types d'apprenants en jouant le rôle de témoin. Ces différentes approches, comme les récits de vie et les biographies langagières, peuvent être englobées au sein d'un Portfolio contextualisé. Pour rappel, les Portfolios Européens des Langues s'articulent autour de trois parties, à savoir :

- une biographie langagière et un passeport des langues permettant aux apprenants d'y répertorier leurs connaissances, expériences linguistiques et culturelles.

- une grille d'auto-évaluation incluse dans la biographie langagière les invitant à réfléchir à leurs apprentissages et aux stratégies qu'ils peuvent mettre en place.

- un dossier personnel dans lequel l'utilisateur peut consigner des travaux.

Cette structure est, bien entendu, à faire évoluer dans le cadre d'un Portfolio contextualisé car, comme son nom l'indique, le but est de l'adapter en fonction du publiccible. Dans notre contexte d'utilisation en tant que formatrice travaillant dans le milieu associatif j'ai créé et mis en place plusieurs Portfolios contextualisés depuis l'année 2008-2009 (associations, centre sociaux et organisme de formation). Mon statut de formatrice m'a permis de réaliser une immersion totale au sein de ce milieu, de pouvoir faire un bilan sur le contenu de l'apprentissage avec les besoins langagiers des apprenants et les objectifs des formateurs mis en place pour les réaliser et, d'autre part, pouvoir 
effectuer des entretiens avec les apprenants, ce qui nécessitait d'instaurer une relation de confiance. Le recueil d'observables s'est donc constitué directement au sein des cours par le biais d'un mode d'« observation combinatoire» (Razafi, 2008: 168) constitué d'observations participantes, d'entretiens, d'interactions, de transcriptions dans un journal de bord, d'observations empiriques, de reconstructions de données informelles, de productions écrites et orales d'adultes migrants. C'est pourquoi la majorité des observables sont constitués d'interactions groupales ou individuelles animées en classe, recueillies par le biais de divers mediums (audio-visuels, supports écrits, photographiques...) et d'observables non sollicités, c'est-à-dire « des discours produits en dehors du dispositif explicite des observations" (Feussi, 2008: 162) qui permettront d'enclencher et de justifier des entretiens compréhensifs et semi-directifs. Ce mode "d'observation combinatoire » nous a permis de bénéficier d'une meilleure adaptabilité aux conditions de notre terrain d'enquête. En effet, en cumulant ces différentes modalités, nous avons pu, en premier lieu, combler la lacune de ne pouvoir toujours enregistrer en direct les apprenants mais de devoir transcrire a posteriori certaines données qui nous paraissaient intéressantes. Ce ne fut donc pas des entretiens au sens canonique mais recueillis par le biais de stratégies dans le but de laisser les apprenants libres de répondre ou de ne pas répondre aux questions. En cumulant ces différentes modalités, cela nous a donné l'opportunité de mettre en place une stratégie de contournement, qui n'est pourtant pas caractéristique de l'approche biographique, et de pouvoir conserver cette stratégie tout au long de notre enquête. Nous avons aussi utilisé la méthode des commentaires provoqués : «méthode consistant à faire parler des sujets ou des groupes sur des cas spécialement construits pour solliciter leurs réactions et leurs jugements, pour analyser, ensuite, ces "commentaires" en fonction de divers objectifs " (Muccielli, 1996 : 27). Cette dernière méthode, que nous avons adaptée en confrontant les apprenants à leurs propres commentaires, présente l'intérêt d'être une instance de vérification des données précédemment recueillies. Les apprenants, en revenant sur leurs commentaires, peuvent les compléter en donnant davantage de précisions ou éventuellement les modifier. Dès lors, cette méthode permet d'aller vers l'approfondissement des observables, ce qui est caractéristique de la méthodologie qualitative. Au niveau de la construction et de l'analyse de ces observables, nous avons tenté d'engager et d'alimenter avec nos apprenants, des entretiens qui se rapprochent de la conversation. La grille, dans le cadre des entretiens compréhensifs, est très souple et nous avons fait le choix d'une grille d'entretien basée sur une progression thématique. Certaines parties de ce corpus ont servi à la construction du matériel didactique. Ainsi, les mediums constitueront des supports de recherche et des supports didactiques. L'une des premières activités mises en place, portait sur le spectacle «Pose ta valise $»^{1}$. Ce spectacle fut créé à partir d'ateliers d'écriture sur les thèmes de l'exil, du départ et de l'arrivée. Le fait d'avoir réuni dans ce spectacle théâtral plus de soixante-dix témoignages de femmes constituait pour notre recherche un medium adéquat dans la mesure où les thèmes abordés rejoignaient nos perspectives de recherche. Le titre évocateur du spectacle « Pose ta valise " a effectivement permis d'amorcer la discussion et d'apprendre où chacun des apprenants a pu poser sa valise et les raisons de son départ. À travers le spectacle « Pose ta valise ", qui a servi de point de repère et d'appui à une séance de Portfolio sur les départs et les arrivées, les apprenants se sont progressivement détachés de ce medium pour s'appuyer sur le récit de leur camarade. Il s'est alors créé une sorte d'effet " yoyo ", les apprenants se mettant à établir des liens entre les différents parcours des membres du groupe. L'utilisation de ce spectacle comme medium nous a surtout permis de travailler 
sur les représentations du statut de migrant et de ses difficultés. Les apprenants se sont effectivement rendu compte que leur situation était similaire à celle de beaucoup d'autres personnes : ils ont donc fait le lien entre parcours personnels et histoire collective. Par l'intermédiaire de ce medium, des sentiments comme la déception, la tristesse, la joie, ainsi que les éléments composant leur valise ont émergé. Ces textes restent, pour la plupart, des textes lacunaires, partiels, qui mettent en évidence les événements les plus significatifs, des liens entre leurs trajectoires migratoires incluant des parcours de "socio-autonomisation » et l'apprentissage de la langue française ont pu surgir sachant que je défini le parcours de «socio-autonomisation» (Matalah, 2014 : 75) comme étant le passage d'un état initial (pays de départ) vers une aspiration à l'autonomie dans un état actuel (pays d'arrivée). Cette « aspiration » peut se concrétiser, ou non, dans la phase de ce parcours, et peut ainsi se traduire par de multiples tentatives d'adaptabilité réussies, ou non, menées dans des domaines variés de la vie sociale tels que le domaine du travail, du logement, de la famille. Cela nous a permis d'acquérir une vision partielle sur des trajectoires migratoires qui sont des moyens pour nous de mieux comprendre les motivations et objectifs de leur apprentissage de la langue française. Nous avons donc sélectionné pour ce présent article trois trajectoires migratoires qui, d'une part nous paraissaient les plus développées, et d'autre part, entretenaient des points communs et pouvaient apporter un regard complémentaire sur certaines dimensions de leurs trajectoires.

3 C'est par l'intermédiaire du medium "pose ta valise " que fut déclenché le premier fragment de récit biographique de l'apprenante Sophie ${ }^{2}$. Cette dernière nous a présenté un témoignage de vie, mais surtout un témoignage d'un moment spécifique de l'histoire du Cambodge. Les différentes trajectoires migratoires respectent généralement cinq étapes importantes: les motivations du départ, la décision, le départ, le voyage et les premières difficultés dans le pays d'accueil. Perregaux (2006: 34) synthétise ce processus par le biais d'une trame narrative qui illustre le parcours-type de migration de ces publics. Dans le cas de Sophie, sa trajectoire migratoire suit ces différentes étapes. Tout d'abord, un départ causé par un exil politique, ensuite le départ, que Perregaux intitule l'« événement", puis le voyage (une partie à pied et une autre en avion) et enfin ses premières difficultés en France. La trajectoire de Sophie est essentiellement marquée par un exil politique. Cette apprenante, originaire du Cambodge, est arrivée en France en 1974 fuyant un pays dévasté par la guerre. Une guerre civile entre les Khmers et les Khmers rouges qui a duré jusqu'en 1979. Son départ fut précipité, comme l'illustre l'extrait 1, ce qui a certainement entraîné de graves conséquences psychologiques :

24. S : Nous quand on doit arriver au pays, quand on est arrivé parce qu'en 74 la guerre dans mon pays, personne ... Il n'y a pas de valise, il n'y a que des vêtements sur le corps c'est tout (...)

30. S : La guerre au Cambodge.

31. A : Donc vous n'avez pas eu le temps de faire une valise?

32. S : Non, non, non...

33. A : Vous êtes arrivée avec vos vêtements?

34. S: Oui, parce que bon vous courez avec la valise?! Parce que restez deux mois pour arriver en Thaïlande à cacher dans la forêt...

35. A : D'accord.

36. S : C'est pour ça, euh, nous les pantalons, il y a des gens les pantalons, des grands pantalons comme ça, arrivés en Thaillande, restait que des slips c'est tout rayé.

37. A : Ils se sont déchirés, les vêtements...

38. S : Oui, déchirés...

39. A : Parce qu'en fait du Cambodge, vous êtes allés jusqu'en Thailande? 
40. S : Oui.

41. A : D'accord, vous êtes venue en avion, à pied?

42. S : À pied. (Ibidem)

4 Lorsque les camarades de Sophie évoquent où ils avaient posé leur valise, cet élément n'évoque rien pour elle dans la mesure où, la concernant, elle n'a pas eu le temps de la prendre. Elle a simplement quitté le pays avec ses vêtements qui se sont détériorés au cours de son périple. Le caractère précipité de son départ signale que Sophie n'a pas eu le temps de faire ses adieux à ses proches, aux lieux de son enfance. Par conséquent, elle n'a pas eu l'occasion de préparer son départ dans des conditions sereines ce qui doit constituer le premier choc émotionnel d'une longue série. Cette confrontation en direct au récit douloureux nous a fait réaliser que déclencher des récits de vie, c'est aussi prendre le risque de remuer des souvenirs pénibles à la fois pour l'apprenant mais également pour les auditeurs dans le cas où ils auraient vécu des situations semblables. Or, sans l'utilisation de ce medium, Sophie n'aurait sans doute pas évoqué spontanément son exil politique. D'autant plus que cette dernière évoque les atrocités de la guerre, les meurtres ou plutôt le massacre par les Khmers rouges de familles Khmers entières notamment dans l'extrait 2 :

87. A : C'est quoi la différence entre les Khmers et les Khmers rouges ?

88. S : Les Khmers rouges habitent dans la forêt, ils connaissaient pas la loi c'est quoi, qui voulaient tuer tous les gens qui...

89. A : Étaient dans la ville? C'est ça, en fait?

90. S : Dans la ville, tous les personnes, professeurs, docteurs, tous tuer !

91. A : D'accord. Et du coup, c'est qui a gagné c'est les Khmers ou les khmers rouges?

92. S : À la fin c'est les Khmers qui ont gagné.

93. A : Et vous vous étiez une Khmer et vous vouliez fuir les Khmers rouges, ils tuaient tout le monde ou...

94. S : Oui, ils tuaient tout, toutes les familles entières.

95. A : Cela donc peut-être pour fuir la guerre : on fuit son pays parce que cela va plus. (Ibidem)

5 Cependant, dans le cas de Sophie, le récit partiel de sa trajectoire offre, d'une part, les traces d'une réalité historique et a pour but, d'autre part, de dénoncer le massacre de membres de sa famille. C'est ce qu'elle explique au reste de la classe et à moi-même. Elle tente de restituer son témoignage à travers un vocabulaire simple (distinction des Khmers par ville et campagne) en insistant sur certains points importants, notamment les meurtres injustifiés. Son arrivée en France a été, par conséquent, le résultat d'un parcours migratoire extrêmement difficile et dangereux. Son récit révèle que Sophie était à la fois dans une situation d'exil mais aussi de deuil avec la perte de certains de ses proches. Dès lors, à travers son récit restitué, on ne peut que constater que Sophie a nécessairement subi des conséquences psychologiques dont les séquelles ont influencé ses choix actuels et influenceront ses projets futurs. Cette dimension psychosociale est donc à prendre en compte dans son parcours d'intégration; or, en tant que formatrice, il est difficile de gérer ce récit douloureux qui est déstabilisant pour l'ensemble du groupe. Pourtant, nous pouvons constater que Sophie apporte surtout un témoignage de l'histoire de son pays, de cette guerre sanglante, en nous confiant notamment la violence des Khmers rouges qui tuaient sans hésitation. En effet, outre la difficulté d'adopter une réaction de neutralité face à une telle confidence, il me semble que l'empathie exprimée a favorisé la cohésion de groupe : chaque apprenant écoutait attentivement le récit des autres, voire se sentait proche de leur vécu on constate donc une réelle écoute de la part 
des apprenants qui interagissent avec le témoignage vivant de Sophie créant une attractivité qui permet en parallèle de mener un travail à la fois sur la compréhension orale et l'expression orale. Ainsi, ce travail réflexif en co-exploration revêt un double avantage : tout d'abord, il permet à l'apprenant de réfléchir sur son parcours quant au processus de mise en mots, ensuite il permet d'entrer dans une phase d'apprentissage de la langue. Progressivement, le medium de départ est relayé au second plan, les apprenants ne parlant plus de la trajectoire des autres mais de leur propre trajectoire. En outre, la situation du migrant évolue, son statut actuel ne se réduit donc plus uniquement à celui de l'exilé politique. Sophie était autrefois dans une situation de vulnérabilité mais, ce n'est peut-être plus le cas à présent. En revenant sur son récit, elle prend aussi conscience des progrès qu'elle a accomplis, de son évolution, et réussi à projeter ses objectifs. En effet, lorsque Sophie a commencé à travailler, elle dut mettre rapidement en place des stratégies afin de contourner sa non-maîtrise de la langue, par exemple, comme elle le relate elle-même, en comptant les stations de métro. Sophie souligne les problèmes d'adaptation au mode de vie français : la nourriture, les bus, le travail, la garde de ses enfants. Elle évoque également les stratégies mises en place pour pallier ses lacunes, notamment linguistiques, des stratégies qui deviennent pour les autres apprenants, surtout les primo-arrivants, des conseils pour les premiers temps dans le pays d'accueil. Les parcours migratoires varient d'un apprenant à l'autre, le récit de ces diverses expériences migratoires permet de témoigner aux générations futures des conditions de vie des migrants. Elle nous a livré les détails de son départ, de ses premières difficultés, notamment au niveau de la langue écrite, le «choc» culturel, d'autant plus qu'elle n'a jamais été scolarisée, analphabète dans sa langue première, ce qui a eu des répercussions sur sa vie quotidienne en France, comme en témoigne l'extrait 3 :

101. A : D'accord, ok donc le moment du départ et maintenant on va arriver au moment de l'arrivée. Donc je vais réécrire comme ça je vous le ferai passer la prochaine fois, je vais juste réécrire ce que vous avez dit. Alors au moment de l'arrivée, c'est-à-dire une fois que vous êtes à l'aéroport mais de l'autre côté.

102. N : Là, c'était dur. [Rire]

103. S : C'est vrai quand je suis arrivée à Paris, en France, bon comme nous jamais mis les pieds à Paris, vous savez, le Paris est bien, il est grand, mais quand vous ne parlez pas le mot français, c'est compliqué pour nous, vous n'arrivez pas à trouver les toilettes, après chercher le sel, demander, quand vous ne savez pas lire vous prenez n'importe quoi, vous goûtez, n'importe quoi, c'est vrai! Nous on est comme ça, nous demandons le grand sel en morceaux on ne sait pas si c'est du sel ou c'est pas du sel, on goûtait. Quand chez nous on mettait dans des sacs comme ça, oui c'est du sel, c'est machin... Mais ici, la boîte en carton qui était fermée, on voyait les grains mais on ne sait pas si c'est du sel, quel goût, mais on goûte...

104. A : Vous étiez obligé en fait obligé d'acheter pour voir si c'était du sel ou pas?

105. S : Oui dans les magasins, c'est vrai pour chercher du sel mais nous ce n'est pas parler c'est quoi du sel? ! C'est quoi du sucre? [Rire]

106. A : Non mais c'est vrai.

107. S: C'est vrai, c'est ça.

108. A : Du coup vous avez fait comment? Du coup, vous avez pu vous débrouiller comment?

109. S: Du coup, arrivés chez nous, nous voyons des grains et puis c'est vrai cela ressemble au sel mais non c'est du produit pour faire le ménage...»

110. A : Non. (Ibidem)

6 Je note, par le biais de ces anecdotes qu'elle rapporte, une certaine frustration, notamment dans la séparation contrainte d'avec son nouveau-né. Elle est aujourd'hui capable de procéder à une certaine prise de recul, une prise de distance vis-à-vis de son 
vécu qui fut parsemé de situations d'incompréhension profonde. Avant même le début de son parcours de "socio-autonomisation", je constate que, dans sa trajectoire, les premières difficultés dans le pays d'accueil se cumulent à une expérience migratoire douloureuse.

Dans le cas de Fathia, le second exemple de trajectoire migratoire, l'absence d'expérience professionnelle a eu des conséquences sur son parcours de "socio-autonomisation » depuis son arrivée en France il y a dix ans. Fathia, originaire d'Algérie, avait 49 ans au moment de l'entretien. Elle a, au cours de l'entretien, fait le choix de s'exprimer en arabe, estimant que son niveau oral en français était insuffisant. Par ailleurs, au cours de l'interaction, elle utilisera de manière involontaire des mots français arabisés. Ils sont mis en évidence dans les transcriptions par le caractère italique tels que : "changite»; " parlou »; « achetou » ${ }^{3} .$. etc. Cette apprenante, tout comme Sophie, n'a pas été scolarisée, mais elle a reçu un enseignement religieux qui lui a permis de maîtriser la langue arabe à l'écrit. Toutefois, elle garde de mauvais souvenirs de cet apprentissage, liés aux conditions difficiles de travail dans des installations précaires comme en témoigne l'extrait $1:$ «Il y a eu une vague de pluie dans notre village, les maisons se sont détruites et cela a détérioré la mosquée, je suis donc allée, changite, dans une autre mosquée » ( Ibidem). Cet enseignement religieux fut brutalement interrompu avant l'adolescence, ce dont elle conserve une certaine frustration. Bien que l'apprenante soit lucide quant à l'influence de son âge sur sa mémoire, elle accuse cependant cette dernière d'être l'origine de tous ses maux : elle éprouve des difficultés à retenir les éléments travaillés au sein de l'atelier comme l'illustre un passage de l'entretien extrait 2 :

239. F : Regarde aujourd'hui mon nom je l'ai écrit difficilement, regarde la situation dans laquelle je me trouve. Hum. Quand une personne dans l'âge cela devient très difficile.

240. A : Ce qui est difficile c'est que vous ne mémorisez pas c'est ça?

241. F : Oui, je te l'ai dit l'autre jour, je regarde le tableau, écrire bien, posi question bien après je rentre à la maison perdu. (Ibidem)

Fathia est venue en France dans le cadre d'un regroupement familial. Son apprentissage a été retardé en raison d'une illusion: celle d'un retour en Algérie. En effet, pensant pouvoir y retourner définitivement, son mari n'a pas souhaité lui enseigner le français. Il lui a affirmé vouloir éviter d'encombrer sa tête avec des enseignements qui lui seront par la suite inutiles. Or, ce « retour » envisagé est généralement un retour improbable et une illusion commune à de nombreux immigrés. Suite au décès de son mari, elle n'envisagera plus de retour définitif en Algérie mais cela la conduira à s'isoler d'autant plus qu'elle ne maîtrise pas la langue française. N'ayant pas beaucoup d'amis et de proches en France, elle m'avoue que la principale difficulté qu'elle rencontre est la solitude : «La difficulté c'est la solitude, la solitude c'est vraiment pénible» (Ibidem). C'est pourquoi cette apprenante est rarement absente en classe, d'une part du fait de l'importance donnée à l'enseignement qui prend un caractère vital comme on le constate dans l'extrait 3 :

183. F : Je me suis accrochée à l'école c'est pour ça.

184. A : Voilà.

185. F : C'est pour cela que je te parle, c'est obligé.

186. A : D'accord. Et depuis votre entrée, vous que euh, que euh [silence]

187. F : Quand j'apprends quelque chose je rentre heureuse à la maison je te jure.

Hum, hum, je te jure heureuse à la maison. (Ibidem)

Et d'autre part pour le caractère socialisant des cours, qui constitue, pour elle, l'une de ses rares sorties, lui donnant l'occasion de s'ouvrir aux autres, à une autre culture, tout 
en progressant. Les cours lui offre, comme elle le déclare, une ouverture sur le monde extérieur. L'élément déclencheur l'incitant à pousser la porte de la salle de classe a été un entretien avec une assistante sociale : "Même l'assistance sociale m'a dit: "Madame, va à l'école et étudiez car tu viens me dire deux, trois mots et tu me regardes, ce n'est pas bien. Tout le temps tu m'emmènes quelqu'un qui vient traduire pour toi, apprends c'est mieux pour toi." » (Ibidem)

En effet, Fathia, ayant perdu son mari, s'est senti contrainte d'entrer en apprentissage par les encouragements d'une assistante sociale qui, un jour, s'est irritée de la voir dépendante des autres, comme cette dernière l'explique dans ce dernier extrait. Pour l'apprenante, la motivation était essentiellement d'accéder à l'indépendance, une aspiration à l'autonomie que l'on retrouve également chez Sophie. Mais concernant Fathia, elle insiste particulièrement sur cette volonté d'être capable de mener seule les tâches de sa vie quotidienne, du fait qu'elle soit isolée certaines choses sont, pour elle, difficiles à effectuer comme se rendre à la banque, aller chez le médecin, faire les courses, autant de tâches qui lui permettent dans le même temps de pratiquer le français. Au départ, elle était tributaire de son mari, qu'elle suivait partout et en permanence, comme elle le relate. À son décès, elle se retrouve seule, complètement démunie, devant affronter le monde extérieur; elle éprouve alors de nombreuses difficultés, comme elle nous le confie. Ainsi, elle a conscience que la maîtrise de la langue peut lui permettre de s'intégrer, ou du moins, de pouvoir vivre plus facilement au quotidien. Fathia, comme certainement de nombreux adultes migrants, ne ménage pas ses efforts pour « franchir le mur de la honte et surmonter les craintes angoissantes d'un nouvel échec » (Stercq, 1994 : 45). Heureusement, certains apprenants parviennent à surmonter un grand nombre d'obstacles comme en témoigne la détermination de Tassadit.

11 Le troisième exemple de trajectoire migratoire est celui de Tassadit. C'est une femme âgée de trente-neuf ans, également d'origine algérienne, comme Fathia. Elle est plus précisément Kabyle. Tassadit, en France depuis quatre ans, est une primo-arrivante de niveau A.1 en français. Elle assiste aux ateliers depuis trois ans de manière irrégulière. Le critère du temps de présence en France n'est pas toujours significatif du niveau de l'apprenant; en effet, Fathia est en France depuis dix ans, Sophie depuis trente-sept ans. En revanche, un critère probablement déterminant est celui de l'entrée dans le monde du travail comme c'est le cas de Sophie. Pour Tassadit, il s'agit du critère de la scolarisation. En effet, Tassadit fut scolarisée jusqu'à l'équivalent du collège, ce qui lui a permis d'apprendre le français et l'arabe. Par ailleurs, on relève dans sa biographie langagière qu'étant d'origine kabyle, elle a pris l'habitude de parler deux langues : l'arabe dialectal algérien et le kabyle, ce qui peut constituer un élément positif dans son apprentissage. L'entrée en France s'explique, comme dans le cas de Fathia, par un regroupement familial, mené par un mari sur lequel elle s'appuie et dont elle est encore dépendante : « Voilà je débrouille c'est... Si c'est loin je vais avec mon mari ». L'arrivée en France ne se fait pas sans embûches ni sans tension : l'absence de la famille est un élément qui revient souvent dans son discours. Elle le mentionne ici à deux reprises :

99. A : C'est-à-dire la même mais à quel niveau par exemple? Par rapport à

l'Algérie ? Qu'est-ce qui est bien des points positifs et qu'est-ce qui... ?

100. $\mathrm{T}$ : Ici c'est bien mais pas de famille. [Rire]

101. A : C'est la famille qui vous manque?

102. $\mathrm{T}$ : Voilà c'est la famille.

103. A : C'est surtout le euh... Le manque, le soutien de la famille quoi...

104. T : Voilà, voilà c'est la famille...(Ibidem) 
Cette apprenante est mère d'un jeune garçon de trois ans, situation qui la pousse d'autant plus à être confrontée à l'environnement extérieur et donc à communiquer en français, dans le cadre de l'accompagnement de son enfant : dans le domaine de la santé (médecin) et du socio-éducatif (crèche) afin d'exprimer ses symptômes, récupérer son enfant, savoir si la journée à la crèche s'est bien déroulée,...; en d'autres termes, pouvoir mener à bien des interactions classiques de la vie quotidienne. Elle a clairement signalé au cours de l'entretien être l'unique responsable de sa volonté d'apprendre le français : «C'est moi, c'est moi ». L'une des motivations personnelles qui la poussent vers l'apprentissage est de maîtriser l'oral, ne plus parler « avec l'accent » et la lecture : «Parce que je veux parler bien français, je veux écrire bien le français" ou "écrire toute seule ». Concernant Tassadit, mais c'est aussi le cas de Sophie et Fathia, la non-maîtrise de la langue est perçue comme un facteur d'exclusion. Effectivement, pour ces apprenantes, de nombreux problèmes comme le fait de ne pas trouver d'emploi ou toute situation de gêne lors d'un rendez-vous administratif, proviennent de leur non-maîtrise du français. Par conséquent, l'apprentissage de la langue devient facteur de pouvoir qui contribue à surmonter certaines difficultés, liées notamment aux différences culturelles. L'apprenante ressent le désir d'apprendre car cela lui permet d'éviter des situations embarrassantes. La honte de l'apprentissage et la gêne de l'échec entrent en jeu à l'extérieur de l'atelier, comme en témoigne la gêne provoquée par l'intrusion d'un inconnu dans l'atelier au moment de l'entretien : être l'objet d'une moquerie, être montrée du doigt comme étant différente, c'est ce qui caractérise cette gêne : «Voilà, j'ai peur quelqu'un parle avec moi je trompe... voilà » (Ibidem). Cela conduit à la dévalorisation de soi, au sentiment d'être « trop bête ». Certains apprenants reposent donc tous leurs espoirs sur cet apprentissage, ce qui peut me semble-t-il, leur réserver à l'avenir des déceptions dans le cas où certains d'entre eux ne parviendraient pas à surmonter leurs difficultés malgré une bonne maîtrise du français. D'où l'importance, pour la majorité des apprenants, d'être surtout capable de lire et écrire. Comme le souligne Catherine Stercq, cet enjeu devient un défi personnel, un but à atteindre. Le monde de la littérature, du livre se pose selon elle comme un monde mystérieux à décoder, très attirant, mais aussi incontournable pour pouvoir prétendre avoir une formation complète : «La relation de l'homme à l'écrit est loin d'être banale. Elle se définit dans un rapport social et culturel et puise des racines dans le sacré. Écrit, objet symbole, au pouvoir mythique, tel que quand on ne sait pas lire et écrire, non seulement on ne sait rien, mais on n'est rien. Aussi apprendre à lire et à écrire met en jeu l'ensemble des déterminants sociaux et culturels des systèmes de valeurs de l'individu » (STERCQ, 1994 : 57). La motivation principale, au-delà de savoir lire et écrire, est l'accès à l'emploi que permet l'apprentissage, ce qui constitue un bien précieux pour Tassadit. En effet, cette dernière voit dans le fait de se rendre à l'école, une action similaire à celle de se rendre au travail, signe que sa vie change, lui permettant d'acquérir un nouveau positionnement. Mais avant même d'atteindre cette étape, constat valable chez la majorité des apprenants concernés, Tassadit possède une compréhension orale relativement bien maîtrisée de la plupart des énoncés, a fortiori s'ils sont émis avec un débit modéré : «Je comprends quelqu'un parle comme ça avec moi tout doucement je comprends mais quelqu'un vite fait comme ça je comprends pas des mots» (Ibidem). Toutefois, on relève un fossé par rapport à l'expression orale, comme l'illustrent si bien les propos de l'apprenante au sujet de sa communication avec son époux : « Des fois, lui il parle en français mais moi je réponds en kabyle » (Ibidem). Venir apprendre le français, ne pas savoir lire et écrire ou ne pas être allée à l'école génèrent des sentiments de honte chez certains adultes migrants. Ces derniers intègrent l'atelier de façon discrète. Certains 
sont gênés par rapport à leurs camarades, ce qui se traduit chez Tassadit par un sentiment d'être impuissante, complètement désarmée face à l'incompréhensible : "Des fois je reste comme ça... Je comprends pas... [rire] Voilà, toujours...» (Ibidem). Bien heureusement, l'apprentissage en cours porte ses fruits grâce à une application immédiate dans la vie quotidienne et des résultats rapides.

\section{Conclusions}

L'un des points qui surgissent de ces différents entretiens est le caractère instable de l'enseignement : ces apprenantes ont toutes connu plusieurs formateurs. Par conséquent, chaque année, un délai de mise en confiance est nécessaire, il faut procéder à une nouvelle identification des besoins, etc. D'où l'intérêt de conserver une trace écrite de leur parcours et de leur progression. Cela permettrait d'éviter à chaque nouveau formateur de partir d'une feuille blanche et d'avoir à sa disposition un certain nombre d'éléments concernant le niveau de l'apprenant, ses difficultés ciblées, les objectifs déjà travaillés, etc. Par ailleurs, apparaît une motivation très forte de la part de ces apprenants, qui se caractérise par leur envie de progresser. Leurs objectifs d'apprentissage sont liés à un désir d'améliorer leur quotidien, de devenir autonomes, ce qui témoigne de leur volonté de s'intégrer dans la société d'accueil. La motivation peut être d'ordre psychologique, suite aux bouleversements opérés quant aux rôles au sein de la famille, notamment lorsque les enfants servent de soutien aux parents. Leur isolement conduit à un manque d'autonomie, une dépendance, comme l'ont affirmé certaines apprenantes à l'égard de leur mari ou de leurs enfants. Cela révèle que le découragement a souvent fait obstacle dans leurs trajectoires et a généré, comme dans le cas de Fathia, un contretemps dans l'apprentissage. Rappelons que cette dernière estimait que l'apprentissage était inutile, étant donnée l'absence de projet de construire son avenir en France. Ces entretiens restent des discours en suspens, synonymes d'une certaine pudeur mais ont tous le mérite de dévoiler la banalité quotidienne de femmes et d'hommes déracinés dont les trajectoires personnelles sont généralement occultées. Or, il ne peut y avoir intégration sans cette prise en compte du migrant dans son intégralité de la part des formateurs et des institutions. En effet, celui-ci, en entrant dans le pays d'accueil, pénètre dans un espace méconnu qui demande un temps d'adaptation; il subit parfois, comme Sophie, des pertes de repères et d'identité. Ce temps d'adaptation peut se traduire, notamment, par un manque de reconnaissance qui peut concerner les expériences passées, les compétences, les diplômes, les langues et qui débouche sur une dévalorisation de soi pouvant aboutir à des blocages psychologiques. Par ailleurs, un autre problème lié à cette non-prise en compte des spécificités du migrant est que le sentiment d'infériorisation peut se transmettre sur plusieurs générations. Dans cette même logique, le Conseil de l'Europe prône les effets favorables du plurilinguisme, mais est-ce réellement mis en place sur le terrain? Ne s'agit-il pas ici d'un "consensus de surface », bien éloignées des réalités ? Cela pose la question de la mise en pratique du plurilinguisme, notamment en proposant des outils concrets à exploiter en classe. S'ajoute à cela un manque de confiance en soi de la part des apprenants, entretenu par la politique d'accueil ; par exemple, dans le cadre du CAI, il n'est nullement question des répertoires langagiers des migrants dans la mesure où ce contrat est le reflet d'une politique monolingue et républicaine. Au contraire, la reconnaissance des spécificités du migrant est le meilleur moyen de ne pas le stigmatiser et de faciliter son intégration. 
Ainsi, le migrant n'est pas appréhendé selon ses lacunes mais selon la globalité, la complexité et la richesse de son parcours langagier, professionnel, familial. L'image du migrant devrait être évolutive, plurielle, riche en nuances et non pas figée selon des stigmatisations et des dépréciations sociales conduisant à des blocages linguistiques. Face au dépaysement dû au passage d'un modèle culturel à un autre, les difficultés de l'apprentissage conduisent nos apprenants vers un ennui profond et poussent parfois vers l'isolement. Toutefois, le «choc » culturel est à nuancer dans la mesure où la distance culturelle s'amoindrit grâce à l'accessibilité de certains médias tels qu'internet et la télévision. Ainsi, la formation en milieu associatif est fortement marquée par cette « visée insertion sociale " qui est encore aujourd'hui profondément ancrée au sein de la formation des migrants. Cette formation se décline sous différents aspects selon les organismes associatifs. Certains ont tendance à privilégier l'utilitaire, le «fonctionnel », c'est-à-dire les besoins immédiats, à un travail linguistique plus solide. Pourtant, ces deux visées gagneraient à être travaillées simultanément. C'est pourquoi, a découlé l'idée de l'initiation des publics migrants adultes en milieu associatif, aux approches biographiques. Ce type de démarche peut comporter une dimension psychologique afin de travailler sur certains blocages comme ceux liés à un exil politique (cas de la trajectoire migratoire de Sophie) et relever un certain nombre de défis didactiques. Ces défis doivent donc prendre en compte les langues et les cultures de chaque apprenant, de leur diversité, en d'autres termes favoriser l'acceptation de soi et l'affirmation des capacités. Les approches biographiques s'ouvrent sur un champ pluridisciplinaire en sciences humaines et sociales qui permettent d'obtenir des clefs d'interprétation (Perregaux, 2002: 82) qu'il s'agira pour moi de transposer dans le domaine de la formation de migrants adultes en milieu associatif et identifier dans quelle mesure de nouveaux modes de socialisation peuvent ainsi émerger. On peut constater qu'elles ont valeur de témoignage historique et social, qu'elles permettent de mieux connaître les apprenants (difficultés rencontrées, objectifs, parcours scolaire...), les influences de l'environnement extérieur, pour mieux identifier leur besoin et les aider à s'autonomiser dans les domaines de leur choix : le migrant n'est pas appréhendé selon ses lacunes mais selon la globalité, la complexité et la richesse de son parcours langagier, professionnel, familial. Faire des retours en arrière peut donc permettre de mieux mesurer son évolution, ses progrès. Nous tentons de mettre en évidence que les démarches biographiques peuvent avoir pour objectif de verbaliser les implicites qui peuvent constituer autant de blocages à l'appropriation des langues. Or, ces non-dits constituent des représentations inconscientes des apprenants et l'un des intérêts des démarches biographiques est en l'occurrence de cibler et d'agir sur ces représentations, permettant notamment de défiger celles liées à l'apprentissage. Les conséquences directes en sont la relativisation des différences culturelles, la promotion de l'intercompréhension et finalement la contribution à l'amélioration de la pratique du français. Cette ouverture sur une pédagogie de la conscientisation de l'apprenant se fait en binôme, dans la mesure où il s'agit d'un outil d'enseignement, un intermédiaire entre le formateur et l'« apprenant » : tous les deux peuvent donc faire évoluer leurs représentations vis-à-vis de l'apprentissage et de leurs pratiques de formateurs ou d'apprenant. Dès lors, les démarches biographiques seraient des supports au profit du formateur et/ou chercheur, de l'apprenant et entre les apprenants mais cette réflexivité ne se limite pas au binôme apprenant-formateur : rien ne nous retient de tenter de faire évoluer les représentations des institutions et décisionnaires car la reconnaissance devrait être réciproque afin de légitimer la position de l'adulte migrant comme sujet plurilingue. Les trajectoires 
migratoires ne peuvent peut-être pas surmonter les blocages mais peuvent permettre de les repérer afin de mieux se projeter dans des solutions et des projets d'avenir notamment dans le monde professionnel. Cette "socio-autonomisation" revêt une dimension identitaire qui recouvre aussi le parcours scolaire, les expériences professionnelles, personnelles, les séjours linguistiques mais également les objectifs futurs tant au niveau de l'apprentissage linguistique que professionnel de l'apprenant. Tout ce qui se rattache à la vie passée/à l'historicité de l'apprenant et qui explique ses choix à venir peut être mis en valeur par l'intermédiaire des démarches biographiques. Il serait donc fécond de créer des passerelles entre différentes dimensions de ce parcours langagier, scolaire et social.

\section{BIBLIOGRAPHIE}

FEUSSI, Valentin (2006). Une construction du français à Douala - Cameroun. Thèse de doctorat. Tours : Université François Rabelais.

MATALAH, Abla (2014). « Enjeux culturels des démarches biographiques auprès des publics migrants en milieu associatif », TraverSCE, $n^{\circ} 15$, pp. 69-81.

MUCCHIELLI, Alex (2009). Dictionnaire des méthodes qualitatives en sciences humaines et sociales. Paris : Armand Colin.

PERREGAUX, Christiane (2002). «Auto-biographies langagières en formation et à l'école : pour une autre compréhension du rapport aux langues », Vals Asla, nº 76, pp. 81-94.

PERREGAUX, Christiane (2006). « Autobiographies croisées : la décentration libératrice d'une lectrice bilingue ", Français dans le Monde - Recherches et applications, nº 39, pp. 31-41.

RAZAFIMANDIMBIMANANA, Elatiana (2008). Langues, représentations et intersubjectivités plurielles : une recherche ethno-sociolinguistique située avec des enfants migrants plurilingues en classe d'accueil à Montréal. Thèse de doctorat. Rennes : Université de Rennes 2.

STERCQ, Catherine (1994). Alphabétisation et insertion socio-professionnelle. Bruxelles : De Boeck Université.

\section{NOTES}

1. Spectacle crée par la compagnie Théâtre du Grabuge [disponible le 20/05/2015] <URL : http://www.theatredugrabuge.com/valise.php>.

2. Transcriptions des entretiens recueillis pendant un travail sur la biographie langagière, Association Rib, 2010.

3. Transcriptions des entretiens recueillis pendant un travail sur la biographie langagière, Centre Social de Pierre Bénite (Lyon, France), 2010. 


\section{RÉSUMÉS}

Cet article porte sur la rencontre d'adultes migrants évoluant en milieu associatif avec les démarches biographiques par l'intermédiaire d'études de cas. L'enjeu de ce travail est de développer la dimension biographique, c'est-à-dire de partir du connu et de leur vécu pour aller vers l'inconnu, potentiellement le moins sécurisant. Et cela, en multipliant l'application en classe de différentes démarches biographiques comme le Portfolio contextualisé, qui permet à l'adulte migrant de progresser dans son apprentissage, d'acquérir des savoirs de base dans la vie quotidienne. Après avoir présenté quelques données importantes quant au cadre institutionnel de ce travail dans le but de mieux comprendre les enjeux politiques, sociaux et didactiques du terrain d'intervention, j'expliciterai, à travers quelques exemples, les enjeux culturels de ces démarches biographiques. Je tenterai de dégager les enjeux réflexifs des approches biographiques notamment les liens entre les trajectoires migratoires incluant des parcours de «socio-autonomisation » et l'apprentissage de la langue française au sein de ces ateliers.

This article concerns the meeting of migrant adults evolving in local associations with biographical researches through case studies. The stake in this work will thus be to develop the biographical dimension, that is to leave the known, their real-life experience, to go towards the unknown, potentially the least reassuring. This will be done by multiplying the application in class of various biographical researches such as the contextualized Portfolio which allow the migrant adult to progress in his learning process, to acquire basic knowledge in his everyday life. Having presented some important data for the institutional frame of this work with the aim of better understanding the political, social and didactic stakes in the ground of intervention, I shall clarify through some examples the cultural issues in these biographical researches. I will try to release the reflexive challenges of the biographical approaches in particular to clarify the links between the migratory trajectories including of the courses of "socio- empowerment" and the training of the French language within these workshops.

\section{INDEX}

Keywords : biographical researches, migratory paths, multilingual socialization, local associations, appropriation of languages

Mots-clés : démarches biographiques, trajectoires migratoires, socialisation, milieu associatif, appropriation des langues

\section{AUTEUR}

\section{ABLA MATALAH}

Laboratoire CERIEC

abla69100[at]hotmail.fr 